\title{
Real Structure of Milled Inconel 738LC Turbine Blades
}

\author{
PALA Zdenek ${ }^{1, a^{*}}$, KOLAŘÍK Kamil ${ }^{2, b}$, BERÁNEK Libor ${ }^{3, c}$, ČAPEK Jiříi ${ }^{4, d}$ \\ KYNCL Jiří ${ }^{5, \mathrm{e}}$, MUŠÁLEK Radek ${ }^{6, \mathrm{f}}$, GANEV Nikolaj ${ }^{7, g}$ \\ ${ }^{1,4,7}$ Faculty of Nuclear Sciences and Physical Engineering, Czech Technical University in Prague, \\ Trojanova 13, 12000 Prague, Czech Republic \\ ${ }^{2}$ Faculty of Mechanical Engineering, University of West Bohemia, Univerzitní 8, 30614 Pilsen, \\ Czech Republic \\ ${ }^{3,5}$ Faculty of Mechanical Engineering, Czech Technical University in Prague, Technická 4, 16607 \\ Prague, Czech Republic \\ ${ }^{6}$ Institute of Plasma Physics, Za Slovankou 3, 18200 Prague, CZ \\ azdenek.pala@fjfi.cvut.cz, ${ }^{\mathrm{b}}$ kamil.kolarik@email.cz, ${ }^{\mathrm{c}}$ libor.beranek@fs.cvut.cz, \\ dcapekjir@fjfi.cvut.cz, ${ }^{\mathrm{d} j i r i . k y n c l @ f s . c v u t . c z, ~}{ }^{\mathrm{f}}$ musalek@ipp.cas.cz, ${ }^{9}$ nikolaj.ganev@fjfi.cvut.cz
}

Keywords: nickel super-alloys, turbine blades, milling

\begin{abstract}
Machining of nickel super-alloys is difficult since mechanical hardening and, consequently, extreme tool wear occur. In the case of turbine blades, castings can no longer meet stringent requirements for precision; hence, they are being ground or milled in order to comply with the demanded dimensions. In this contribution, castings made of Inconel 738LC were machined by four axis milling. The resulting surface of gas turbine blades are characterized by several surface integrity parameters with respect to the structural inhomogeneities caused by mutual effect of plastic deformations and thermal fields during the cutting process. Moreover, the impact of effective contact area of the cutter was considered as well. It will be shown that ideal machining parameters viewed from the standpoint of surface roughness and economy of the whole machining process can lead to appreciable tensile normal stresses.
\end{abstract}

\section{Introduction}

Turbine blade represents a crucial product of today's world and its durability, reliability, fatigue life, or simply quality, have far reaching consequences for areas ranging from power generation to aircraft safety. The very production of turbine blades should, therefore, epitomize the state-of-the-art of the manufacturing industry beginning with the choice of material, through machining processes and ending with surface treatment(s). Nickel super-alloys are an important group of materials used for gas turbine blades because of their heat resistance and favorable mechanical properties such as high strengths at elevated temperatures. These features stem from complicated microstructure with interplay between $\gamma$ and $\gamma^{\prime}$ nickel phases, various carbides and the real structure of individual crystalline phases that is distinguished by a complex character [1] of grain boundaries, presence of defects such as vacations, dislocations, stacking faults and residual stresses (RS).

Being parts of dynamically loaded components operating under non-ambient conditions, the spatial distribution of macroscopic RS and even microstresses in turbine blades substantially affect the fatigue life and contribute either to the service life prolongation or shortening when crack propagation is advantaged by existing stresses during service. It would be therefore appropriate to determine the stresses in the blade after the final manufacturing step and upon modelling the dynamic load, when superposition of residual and load stresses takes place, decide how influential or even critical the RS can be and whether it is, thus, desirable to change the production parameters.

Nevertheless, the microstructure of Ni-based super-alloys is commonly such that two morphologically different crystalline phases with identical space group, i.e. Fm-3m, and very small lattice mismatch [2] are present. One is denoted as $\gamma$ phase and represents the continuous matric of fcc austenite and the other is major precipitation $\gamma^{\prime}$ phase. The tiny lattice mismatch $\delta=2\left(a_{\gamma}\right.$ - 
$\left.a_{\gamma^{\prime}}\right) /\left(a_{\gamma}+a_{\gamma^{\prime}}\right)$ is in the order of $0 . x \%$ and makes the two phases virtually non-distinguishable [3] by powder X-ray diffraction in the case of polycrystalline character of turbine blade surface. A possible and highly debatable approach employing standard X-ray diffractometers for RS evaluation in materials with $\gamma$ and $\gamma^{\prime}$ phases would lie in measuring the diffraction profile, which is the result of diffraction profiles overlapping of both phases, with radiation that gives a sufficiently strong diffraction profile for high two theta angles. It turned out that X-ray tube with manganese anode is the optimal choice in this case, since diffraction line $\{311\}$ of $\gamma / \gamma^{\prime}$ is at approx. $152^{\circ} 2 \theta$.

The challenging step of turbine blade machining is the consequence of rising requirements for precision which the castings can no longer meet. One option for the final machining is grinding, but in the here presented case, the finish grinding process has not delivered the coveted results because it triggered off local melting resulting in presence of undesirable white layer and cracks [4]. On the other hand, the enormous evolution in the material of tools for milling has facilitated substantial reduction in costs due to increase of cutting speed, e.g. up to ten times faster machining with whisker reinforced corundum [5] when compared with traditional cermet WC-Co tool tips. The use of ceramic materials in tool tips represents another benefit of the milling process as their high melting temperature limits the thermally activated tool wear and favors the less harsh abrasive mechanism.

Our aim is to analyse any possible mutual relations between process parameters of four axis milling of casting from Inconel 738LC on one side and surface macroscopic and microscopic RS and grain sizes on the other. Or, simply, find milling parameters leading to purely compressive RS.

\section{Analysed samples}

The analysed turbine blades were machined from Inconel 738LC cuboidal castings with dimension $205 \times 30 \times 30 \mathrm{~mm}^{3}$. The castings were heat treated, but HIP (Hot Isostatic Pressing) was not imposed on them. Hence, the ready-to-be-milled castings included also pores in the structure as seen in Fig. 1. It is needless to say that combination of pores, tensile normal stresses and appreciable shear stresses is expected to be kind for crack growth.

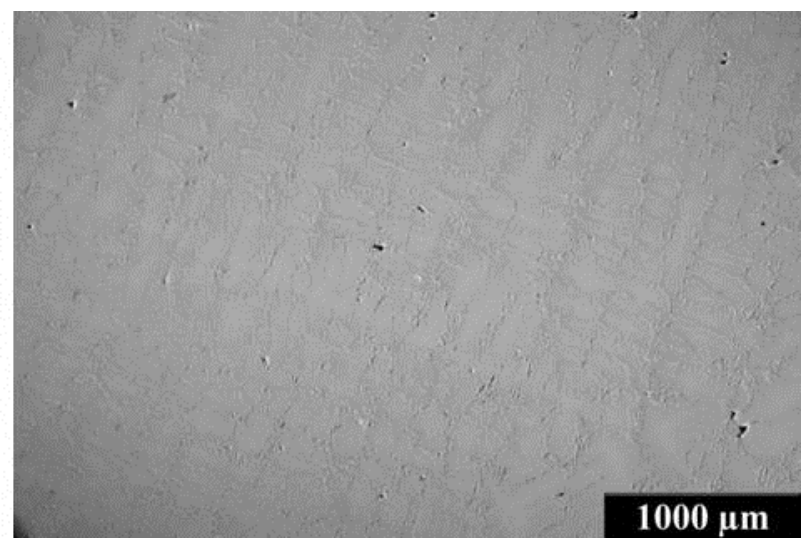

Fig. 1 The characteristic microstructure consisting of $\gamma$ and $\gamma^{\prime}$ phases and pores on the cross-section of the heat treated sample from Inconel 738LC.

Chemical composition of the castings was verified on four arbitrary chosen areas and the result of EDX investigation is in Tab. 1.

Tab. 1 Chemical composition (wt. \%) in 4 areas on the cross-sections of Inconel 738LC.

\begin{tabular}{lcccccccccc} 
Position & Al & Si & Ti & Cr & Co & Ni & Nb & Mo & Ta & W \\
\hline 1 & 3.11 & 0.00 & 3.55 & 16.56 & 9.48 & 61.17 & 0.57 & 1.64 & 0.01 & 3.90 \\
2 & 3.17 & 0.06 & 3.78 & 16.07 & 8.93 & 62.58 & 0.72 & 1.59 & 0.21 & 2.88 \\
3 & 3.19 & 0.10 & 3.98 & 16.60 & 9.41 & 61.21 & 0.76 & 1.81 & 0.07 & 2.88 \\
4 & 3.13 & 0.04 & 3.91 & 17.17 & 9.10 & 60.03 & 0.64 & 1.82 & 0.40 & 3.76
\end{tabular}


Detailed parameters of the four axis milling process cannot be provided because of commercial constraints. However, we used parallel milling with pushed tool and flooding by cooling liquid. The cutter had either 2, 4 or 6 teeth. Tool diameter was $10 \mathrm{~mm}$ and axial depth of cut $0.5 \mathrm{~mm}$. 3D model of the milling process can be seen in Fig. 2. Feed and feed per tooth were variables, altogether 11 combinations of processing parameters were tested with one combination being chosen by the industrial producer as the most convenient from the surface roughness and economy of the whole machining process points of view. The tool tilt in respect to the work-piece $\beta$ was constant and it was set according to our previous investigations of three axis milling of much larger turbine blades [6] when $\beta=28^{\circ}$ lead to the most favorable RS. Yet by choosing this $\beta$, it would be impossible to machine convex areas of the blades. Therefore, new tool suitable for lower $\beta$ was used and the constant value for all milled blades was $14^{\circ}$. During the ensuing XRD investigation, the effective cutting area, or contact zone, was considered as well.



Fig. 2 3D model of the milling process (NX software); the analysed areas by XRD were on one of the edges of the blade and two other were extrema of concave and convex side of the blade. Analysed directions $\mathrm{T}$ (feed direction) and $\mathrm{L}$ are indicated.

\section{Samples characterization}

The XRD measurements were performed on PROTO iXRD COMBO diffractometer in $\omega$ goniometer or iso-inclination mode. As already mentioned, $\mathrm{MnK} \alpha$ radiation was used for diffraction measurements aiming at RS calculations, furthermore, we used $\mathrm{CrK} \alpha$ radiation and vertical $\theta / \theta$ goniometer X'Pert PRO MPD for microstrains and grain size computation and CoK $\alpha$ radiation and $1 \mathrm{D}$ detector for phase composition determination.

The diffraction line $\{311\}$ of $\gamma$ and $\gamma^{\prime}$ phases was measured in order to characterize the macroscopic RS in the blades' surfaces. Values of macroscopic RS in the feed direction $\sigma_{\mathrm{T}}$ and perpendicularly to feed direction $\sigma_{\mathrm{T}}$ were calculated from lattice deformations determined from $2 \theta^{311}\left(\sin ^{2} \psi\right)$ plots following Winholtz-Cohen algorithm [7] and using the X-ray elastic constants $1 / 2 \mathrm{~s}_{2}=6.57 \mathrm{TPa}^{-1}, \mathrm{~s}_{1}=-1.56 \mathrm{TPa}^{-1}$ computed according to Eshelby-Kröner approach [8]. Diffraction angle $2 \theta^{311}$ was taken as a centre of gravity of the $\{311\}$ diffraction doublet MnKa. The presented values of macroscopic RS are arithmetic mean values obtained from 3 measurements. Single-line Voigt function method [9] was applied for calculations of microstrains and average grain sizes using $\{111\}$ diffraction line of $\gamma$ and $\gamma^{\prime}$ phases.

For each gas turbine blade, normal stresses $\sigma_{\mathrm{L}}$ and $\sigma_{\mathrm{T}}$, shear stresses $\tau_{\mathrm{L}}$ and $\tau_{\mathrm{T}}$, microstrains and grain sizes in $L$ and $T$ direction were obtained for three areas; one being on the edge of the blade and the other two in the extremum of concave and convex sides, respectively. These three areas also correspond to three various sizes of cutting zone.

In addition to XRD analyses and microstructural characterization coupled with EDX presented in the preceding chapter, roughness was measured and the shape of the obtained blade checked by laser CMM (Coordinate Measuring Machine) Carl Zeiss Prismo. 


\section{Results}

Phase ID and Rietveld analysis of $\mathrm{CoK} \alpha$ diffraction patterns revealed presence of precipitation phase $\mathrm{Ni}_{3} \mathrm{Al}$ (around 4 wt. \%), less than 1 wt. \% is made up by $\mathrm{AlTi}_{3}$ and carbide with the univalent MC (Metal Carbide) type; the balance formed by $\gamma$ and $\gamma^{\prime}$ phases as seen in Fig. 3. The unsuitable fit of $\{002\} \gamma / \gamma^{\prime}$ diffraction profile (at $60^{\circ} 2 \theta$ ) mirrors the fact that elastic anisotropy was not included in the structure model. The chosen diffraction profile of $\{311\} \gamma / \gamma^{\prime}$ used for RS analyses is for $\mathrm{CoK} \alpha$ at $111^{\circ} 2 \theta$. Diffraction patterns barely changed with milling parameters and detrimental $\sigma$ phase was not observed.

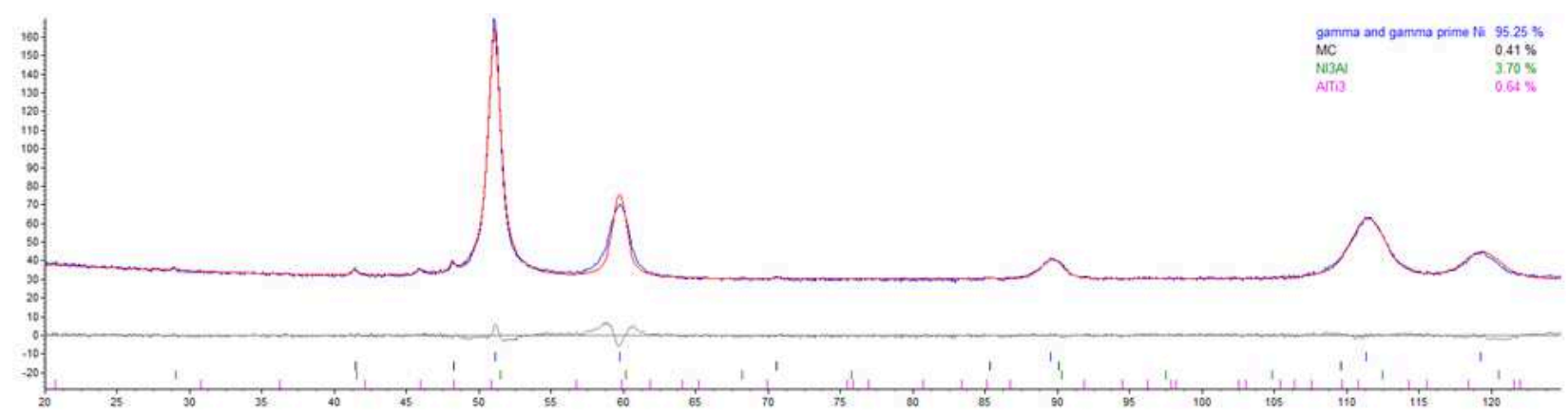

Fig. 3 Result of Rietveld analysis (done in TOPAS 4.2) of representative surface area on gas turbine blade made from Inconel 738LC; $\mathrm{R}_{\mathrm{wp}}=5.55$. Grain size of $\gamma / \gamma^{\prime}$ calculated according to fundamental parameters approach [10] was in the range from 20 to $30 \mathrm{~nm}$.

The blade milled with parameters evaluated as roughness-cum-economy most convenient showed $\mathrm{R}_{\mathrm{a}} \approx 0.5$ and $\mathrm{R}_{\mathrm{z}} \approx 2.3$ and the obtained values of RS are in Tab. 2 . They reveal that in the extremum of convex region, stresses in the direction perpendicular to tool feed can approach $1 \mathrm{GPa}$ which is very close to the Inconel 738LC tensile strength of approx. 1.1 GPa. This "ideal blade" is, thus, far from optimum when RS are included in the considerations. This was a clear argument for process parameters optimization from the standpoint of RS.

Tab. 2 Normal $\sigma$ and shear $\tau$ RS in all six investigated points on the surface of "ideal blade".

\begin{tabular}{|c|c|c|c|}
\hline Direction & Area & $\sigma \pm \Delta \sigma, M P a$ & $\tau \pm \Delta \tau, M P a$ \\
\hline \multirow{3}{*}{$L$} & edge & $-29 \pm 27$ & $-184 \pm 13$ \\
\cline { 2 - 4 } & concave & $506 \pm 42$ & $164 \pm 21$ \\
\cline { 2 - 4 } & convex & $947 \pm 54$ & $1 \pm 27$ \\
\hline \multirow{3}{*}{$T$} & edge & $-392 \pm 27$ & $1 \pm 13$ \\
\cline { 2 - 4 } & concave & $413 \pm 47$ & $105 \pm 23$ \\
\cline { 2 - 4 } & convex & $282 \pm 60$ & $26 \pm 30$ \\
\hline
\end{tabular}

All calculated normal stresses in the tool feed direction $\left(\sigma_{\mathrm{T}}\right)$ and perpendicular to it $\left(\sigma_{\mathrm{L}}\right)$ irrespective of the number of cutter's teeth are summarized in Fig. 4 a, b. Since the $2 \theta^{311}\left(\sin ^{2} \psi\right)$ plots repeatedly exhibited the so called $\psi$ splitting, shear RS $\tau_{\mathrm{T}}$ and $\tau_{\mathrm{L}}$ are displayed as functions of number of the tool's teeth in Fig. 5 a, b regardless of the tool feed value. 

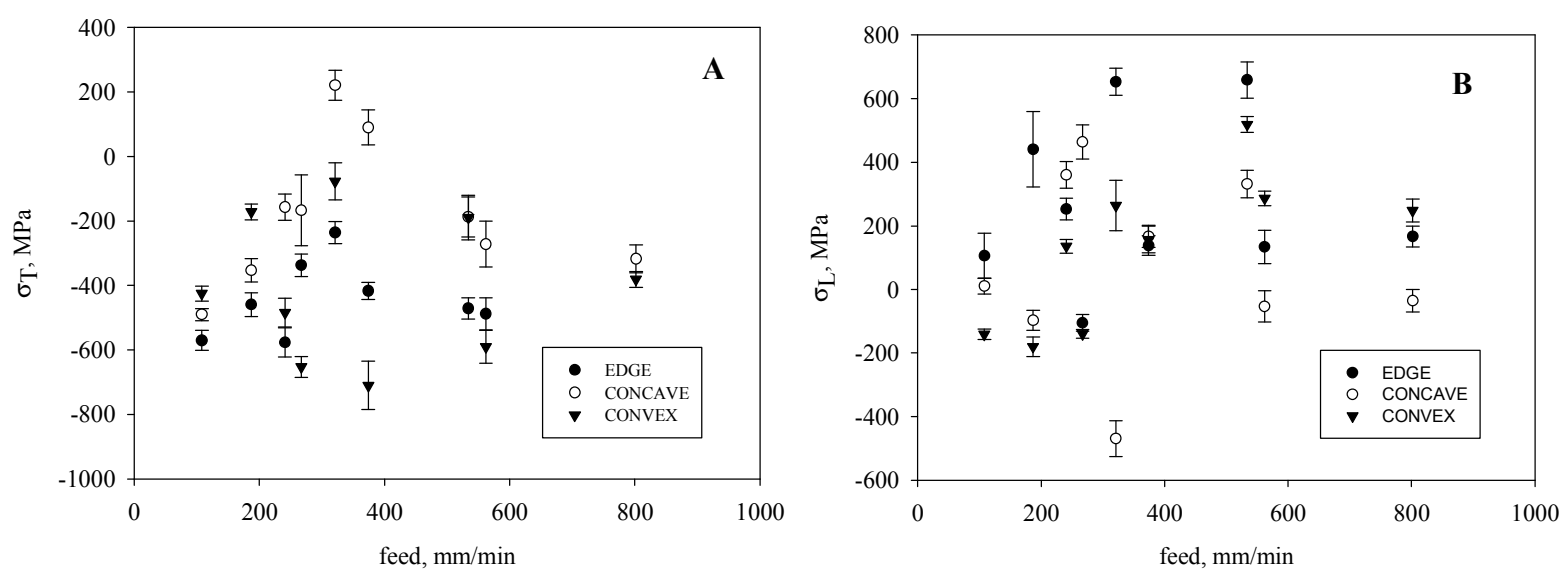

Fig. 4a, b Normal residual stresses in directions of tool feed (A) and perpendicular to it (B).
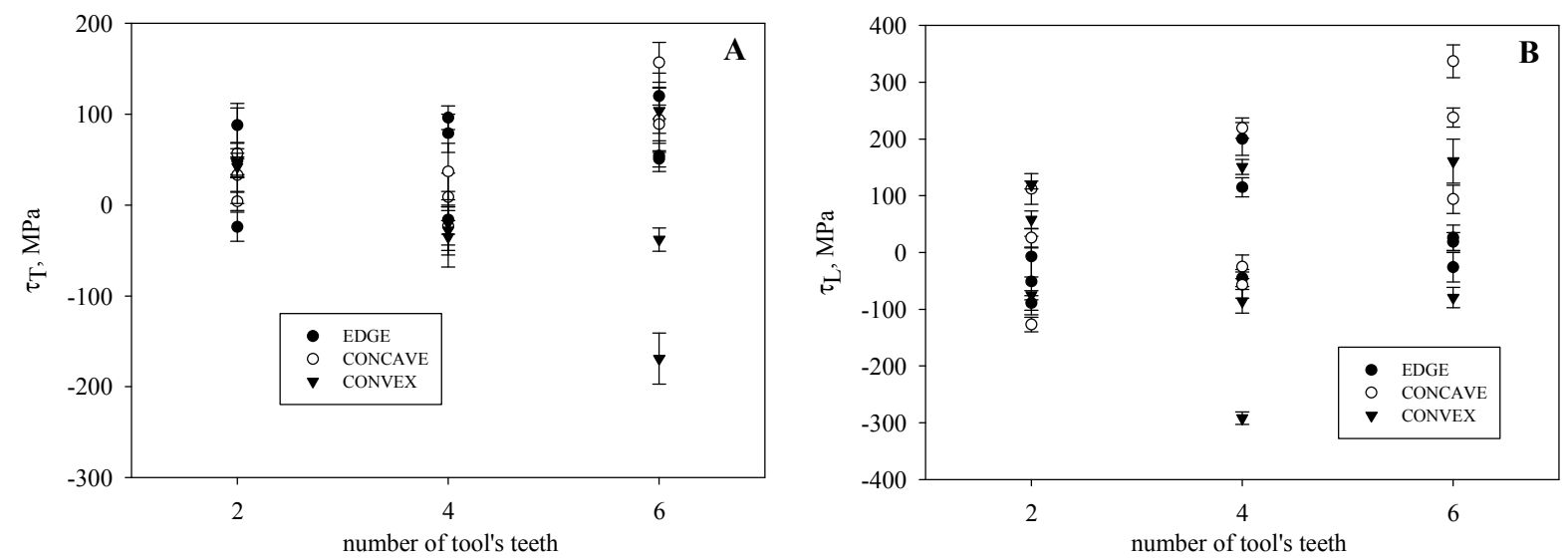

Fig. 5a, b Shear residual stresses in directions of tool feed (A) and perpendicular to it (B).

The sizes of coherently scattering domains evaluated according to single line Voigt function method were in the range from 20 to $30 \mathrm{~nm}$. Values of microstrains lie in the interval from 5 to 6 milistrains, i.e. $5 \div 6 \cdot 10^{-3}$, which would correspond to microstresses in the range from 1.1 to $1.3 \mathrm{GPa}$. In this case, the agreement between fundamental parameters approach implemented in TOPAS 4.2 Rietveld analysis software and results of single line Voigt function method is superb. However, we did not observe any mutual relationship between machining parameters and either grain sizes or microstrains.

\section{Discussion}

Residual stress determination by X-ray diffraction for industrial partners is certainly becoming more commonplace and sought after. However, the requirements sometimes call for solutions beyond the standard XRD methods. This is often the case when residual stresses in polycrystalline nickel super-alloys are of interest. The near to perfect overlap of $\gamma$ and $\gamma^{\prime}$ phases in diffraction pattern does not facilitate determination of residual stresses for either phase separately and, in this contribution, it is suggested that the residual stresses in the sample are characterized by stresses computed from diffraction profile of $\{311\}$ planes of both phases which has maximum at approximately $152^{\circ} 2 \theta$ when MnKa radiation is used.

Following this approach, the task was to find out whether the gas turbine blade milled with ideal parameters in respect to the surface roughness and machining process economy also fulfils certain limits imposed on surface residual stresses. We established that the residual stresses can locally approach tensile strength which called for tuning of milling parameters. The goal was, hence, to find such combination of milling parameters that would lead to compressive RS in both tool feed 
direction and direction perpendicular to feed and, if possible, also establish at least qualitative relationships between e.g. feed or number of cutter's teeth or size of cutting zone, and parameters of real structure. The first goal was fulfilled, the other, sadly, not as there were no pronounced and monotonous relations.

From the performed XRD analyses, the milling parameters leading to compressive RS in both directions and for all sizes of cutting zones, i.e. in the edge and in extrema of convex and concave side of the blade, are when 2 teeth cutter is used and low feed of $108 \mathrm{~mm} / \mathrm{min}$ applied and feed per tooth is $0.04 \mathrm{~mm}$. The compressive RS in feed direction are actually higher than $150 \mathrm{MPa}$.

In general, the macroscopic RS have mostly compressive character in direction of tool feed, see Fig. 4 a,b. In fact, there are only two "tensile exceptions" in this direction. However, both tensile and compressive RS are observed in direction perpendicular to feed. Another issue are shear stresses. The so called $\psi$ splitting was typical for virtually all $2 \theta^{311}\left(\sin ^{2} \psi\right)$ plots and the shear stresses reach startlingly large values, which are up to $100 \mathrm{MPa}$ in both directions for 2 teeth cutter, but in case of 6 teeth cutter amount to even unrealistic $300 \mathrm{MPa}$. Whether this is the consequence of the used approach is hard to assess.

\section{Summary}

It was found that gas turbine blade made from Inconel 738LC super-alloy and milled with parameters ideal form the view of surface roughness and machining process economy can locally approach residual stresses that are close to tensile strength. Ensuing optimization of milling parameters in respect to several real structure parameters with most notable being macroscopic residual stresses lead to conclusions that the favourable compressive RS are generated when 2 teeth cutter is used and low feed of $108 \mathrm{~mm} / \mathrm{min}$ applied and feed per tooth is $0.04 \mathrm{~mm}$. Moreover, the obtained values of shear stresses increased with increasing number of cutter's teeth. There is no pronounced and monotonous dependence between values of normal RS and feed. Yet for the chosen milling conditions, RS in the tool feed direction had dominantly compressive character, but were mostly tensile perpendicularly to feed. Other analysed real structure parameters, i.e. microstrains and grain sizes, do not deviate beyond comparatively narrow intervals of 5 to 6 milistrains and 20 to $30 \mathrm{~nm}$, respectively, and do not exhibit any correlation with the applied range of milling parameters.

Acknowledgments - This research was carried out in the frame of projects NEXLIZ CZ.1.07/2.3.00/30.0038 and TA02011031 (Technology Agency of the Czech Republic).

\section{References}

[1] U. Heubner, Nickel alloys, first ed., CRC Press, 2000.

[2] J. Li, R.P. Wahi, Acta metal. mater. 43 (1995) 507-517.

[3] G. Brunetti, A. Settefrati, A. Hazotte, S. Denis, J.-J. Fundenberger, A. Tidu, E. Bouzy, Micron 43 (2012) 396-406.

[4] M.C. Shaw, Surface Melting in Grinding Operations?, Ann. CIRP 33 (1984) 221-223.

[5] I.A. Choudhury, M.A. El-Baradie, Machinability of nickel-base super alloys: a general review, J. Mat. Proc. Technol. 77 (1998) 278-284.

[6] K. Kolařík, Z. Pala et al., Applied Mechanics and Materials 486 (2014) 9-15.

[7] R.A. Winholtz, J.B. Cohen, Aust. J. Phys. 41 (1988) 189-199.

[8] J.D. Eshelby, Proc. Roy. Soc. A 241 (1957) 376 - 396.

[9] T.H.de Keijser, J.I. Langford, E.J. Mittemeijer, A.B.Vogels, J. Appl. Cryst. 15 (1989) 308-314.

[10]R.W. Cheary, A. Coelho, J. Appl. Cryst. 25 (1992) 109-121. 